\title{
Virtual Reality Immersion: An Important Tool for Diagnostic Analysis and Rehabilitation of People with Disabilities
}

\author{
Helda O. Barros, Marcelo Márcio Soares, Epitácio L. Rolim Filho, \\ Walter Correia, and Fábio Campos \\ Post-graduate program in Design, Universidade Federal de Pernambuco, \\ Recife, Pernambuco, Brazil \\ helda_barros@yahoo.com.br, marcelo2@nlink.com.br, \\ rolimfilho@uol.com.br, ergonomia@terra.com.br, \\ fc2005@gmail.com
}

\begin{abstract}
This paper sets out the case for the importance of using virtual reality immersion for diagnostic analysis and rehabilitating people with disabilities. To do so, a review of the literature was undertaken by examining articles published between 2000 and 2012. The results show that browsing in virtual immersion environments simulates real-world situations, with the advantage that this enables there to be full control over the variables analyzed and consequently over the health aspects involved. Furthermore, when using multisensory channels, the human-task-system interface tools enable there to be simultaneous treatment of multiple morbidities, which characterizes there having been an important advance made in the functional independence of people with disabilities.
\end{abstract}

Keywords: virtual reality, people with disabilities, rehabilitation.

\section{Introduction}

Virtual reality uses an interactive environment, created by software and hardware, so as to give users sensations similar to those in the real world. Virtual environments can be used in any creative process, ranging from the presentation of ideas to the final phase of conceiving projects. [15]

Virtual reality is a way of transporting the subject to a virtual environment in which he/she is not physically inserted, but which generates this sensation [19].

The use of multisensory devices, browsing in three-dimensional spaces, immersion in the context of the application and interaction in real time extend the senses previously linked to simply viewing, listening and manipulating to touching, feeling pressure, and even smelling, and this leads to migrating to the concept of learningknowing-feeling-communicating [4].

In addition, Ergonomics considers technical indicators related to usability, applicability and utility as constructs that are essential to users regarding the interface as comfortable and mindful of their well being [19]. 
This result will be achieved only when users are involved in the cycle of assessing, diagnosing, designing and validating their physical environment, whether in the design of products, of environments or of urban space. A process of User-Centered Design will promote better knowledge of subjects, their needs, expectations and abilities, and thus achieve what projects set out to do [19].

When projects serve specific populations, not only in the social sphere, but by including the user in the perspective of the disability, the creation process attains its most important contribution: this characterizes design as a tool that promotes good health.

Thus, the design process goes beyond the goals of efficiency, effectiveness and satisfaction and achieves a new construct: functionality, which the World Health Organization considers is a variable which is essential for defining the state of health itself.

Within this context, this paper seeks to set out the advantages of using virtual reality in the process of rehabilitating people with disabilities, and stresses the efficiency and effectiveness of the gain in functional independence by users who have been submitted to virtual immersion environments.

\subsection{Rehabilitation in the Virtual Environment}

Currently, virtual reality is being used in several areas of rehabilitation. Tools have been developed for training de-ambulation in patients with Parkinson's disease in order to facilitate a gait pattern. Virtual environments have been used for children with cerebral palsy to improve spatial perception and the operation of motorized wheelchairs. And likewise in the post-operative period of surgeries of the hand and in the rehabilitation of stroke patients [8].

This technology offers the ability to create an environment in which the intensity of the training can be systematically manipulated and individualized, thereby seeking motor learning that is appropriate for each patient by means of semi-automated programs [7].

The objective of the characteristics of hardware and software, and the complexity of the task requested are to provide users with a significant experience in the context of the researcher's therapeutic goals. These include the opportunity for active learning, which encourages and motivates the participant, the ability to measure behavior objectively into a challenge, the security of maintaining control over the experiment and the ability to measure results [5].

The multisensory resources of virtual reality can promote behavioral changes, improve neural plasticity in children and encourage learning and performance [4].

The human forms of apprehension are multimodal and, as regards the constructivist assumptions of learning, this means putting the user at the center of the process, thus giving him/her the tools necessary for experimentation-decision [12].

Learning is an individual and complex process, which is underpinned by the individuals' experiences and by his/her consequent global view of the world [14, 21]. As it is related to the development of the skills of observation, analysis, planning, decision, implementation and evaluation, VR is seen to be a powerful feature, since it prompts visualization, interaction and real-time responses [17]. 
Thus, considering the prognosis and the very evolution of the disease, sensorymotor cognitive learning is founded on pathological bases, and influences the subject's mental models with, in and about the world, thus exacerbating his/her motor and cognitive disabilities and making the planning and carrying out of activities and skills difficult $[2.7,24]$

The formation of mental models is therefore based on sensorimotor engrams ${ }^{1}$ influenced by the physiological changes caused by the disease [18, 23]. Experiments are undergone in abnormal pathological bases which alter the proprioceptive responses of arthresthesia and kinesthesia, and cause considerable postural orientation and balance deficits [16, 20, 22].

In this context, virtual reality immersion, by activating the cognitive processes needed for browsing and interpreting the environments, will promote the automation of daily activities, thus causing permanent changes in subjects' mental models. This is because immersion is characterized by the user's perception of his/her being inserted into a real environment, while being directly linked to the settings of the virtual environment and the sensations of seeing, hearing, touching and feeling that the virtual one causes. [19]

The central nervous system is not able to distinguish between the sensory-motor experiences and relational experiences that occur in the real world and in virtual environments. During immersion, learning overcomes the limit of the cognitive mind supported by sensory-motor corporeality, thus permitting a full analysis of the subject in an environment of controlled variables [20, 22].

The researcher can then define the plans of sensory-motor action and mentalrelational action thus controlling a new process of self-awareness and promoting the alteration of the postural engrams within the pathology itself.

\subsection{Immersion in Virtual Environments}

The concepts of immersion, presence, interaction and involvement are fundamental in order to understand subjects' physical and psychological experiences in virtual environments [19].

Immersion provokes the sensation of living in the real world in users when they are browsing in the virtual environment. On the other hand a feeling of being present is linked to the psychological aspects involved, this being verified in the sensations provoked - visual and auditory ones - in the subject's relationship with the virtual interface [19].

The involvement depends on the concentration of the individual on the virtual environment and on the isolation from the real world, being a better fit for immersion.

Finally, the interaction is about the connection between the user and the virtual environment, and evaluates the efficiency and effectiveness of the communication of this system [10].

${ }^{1}$ Engram is a permanent feature in the cerebral cortex, resulting in learning, training or experience in which the interneuronal synaptic become broader, more secreting neurotransmitters and richer in postsynaptic receptors. 
Immersion VR, the object of this research, depends on artifacts that will allow the sensation of living in a real world to be experienced.

The sensation is so real that sensory conflicts may even cause uncomfortable symptoms after immersion in virtual environments. In this context, fatigue, eyestrain, dizziness, ataxia, visual flashbacks, disorientation and imbalance stand out $[6,9]$.

The resources used in immersion VR are: audio-visual devices, which help the sensation of movement and location; tactile interfaces, which produce sensations of shape, warmth and texture; and kinesthetic response, which requires the use of force, in addition to perceptions of pressure and vibration. [12]

\subsection{Use of Haptic Devices and Their Importance for the Virtual Environment}

Virtual reality and tactile technologies have emerged as promising tools to support the diagnosis of patients and interventions in the rehabilitation process [1].

Besides virtual reality systems, which provide virtual environments in 3-D within which the user can browse, haptic devices improve the user's interactivity and task performance.

Haptic, a term which was derived from the Greek verb "Haptesthai" which means "touch", adds the sense of touch and feedback of forcevin human-computer interaction.

Haptic devices enable the user to manipulate objects in virtual environments in a natural and effective way and can provide information on matters such as the rigidity, texture and weight of objects [11].

In rehabilitation, the objective is to help people with disabilities to improve their functional independence, by recovering their strength and range of movements. By helping patients improve their motor skills, thereby compensating for the permanent loss of function, patients can achieve a better quality of life.

Due to the feedback of force, tactile devices are indispensable tools in virtual rehabilitation because they help to measure performance and adjust the basis of exercises for each patient [25].

This potential for evaluating the patient's performance by measuring different parameters, which cannot be evaluated in traditional rehabilitation, may be of benefit to patients and professional therapists.

\section{$2 \quad$ Methodology}

The study consists of a review of the literature which fits into the presentation of papers in the category of theory.

First of all, 107 papers were pre-selected by conducting a search that used keywords. The search placed emphasis on the areas of Ergonomics and Health - namely: Applied Ergonomics, Ergonomics, Human Factors and Ergonomics and International Journal of Industrial Ergonomics, and on the databases of IEEE, Scielo, Pubmed and Medline. 
In addition to journals, the research also included the annual IEEE International Conferences on Virtual Reality held between 2009 and 2012.

After analysis, only 25 publications were used to draw up this article. The selection of articles was based on their appropriateness to the research context and the impact factor and the periodicity of the journals.

\section{Discussion}

The virtual environment offers opportunities to manipulate therapeutically relevant variables and to work on the performance of motor and cognitive skills during immersion. The rehabilitation of each deficiency will depend on the movements required, the number of repetitions, the length of the experiment, the speed of presentation of the stimulus and of the complexity of browsing the environment itself, while always observing the time required to perform the task [6] .

The main advantages of virtual rehabilitation are repetition, feedback on performance and motivation. Moreover, recent studies with stroke patients $[5,7,8]$ have proved that VR can contribute positively to the neural organization and recovery of functional motor skills.

The largest effect of immersion is the perception of presence in the virtual environment. The sense of reality enables a larger number of neural units to be recruited, thus constituting in addition to the motor stimulus, an important item of cognitive training.

On the other hand, the greatest difficulty lies in the very design of the virtual environment. First of all, users' needs and functional limitations must be listed, based on the User-Centered Design method, which includes a prospective clinical study of a representative sample. Later, the design should limit the deficiencies that will be addressed, and list the main inabilities and the correlated symptoms and clinical signs for each disability, thus determining the interfaces to be used in the virtual environment.

In order to construct the virtual environment, the resources used in immersion are: audio-visual devices, which help the sensation of movement and location; tactile interfaces, which produce sensations of shape, warmth and texture; and the kinesthetic response, which requires the use of strength, besides the perceptions of pressure and vibration.

It is important to emphasize that, within the concept of Inclusive Design, the virtual environment of rehabilitation should cover the broad spectrum of disabilities. This concept is essential for the rehabilitation of multiple functional variables. However, this makes the design of the virtual environment even more complex.

The use of virtual reality as a tool for diagnostic analysis and rehabilitation is undoubtedly a trend. However, studies are still scarce and their sample sizes small. Moreover, there is no reference standard [13].

So that a discussion of different research studies may be reliable, the following variables need to be considered: sample size, age range, gender and ethnic characteristics, type of disability and length of sequel. This is because the same functional limitation can be caused by different deficiencies. 


\section{Conclusion}

Making use of virtual reality enables real-world situations to be simulated, thereby giving the researcher total control over the variables analyzed and enabling the health aspects involved to be controlled.

The simultaneous control of variables is of the utmost importance in the systemic treatment of the disease, as it allows patients with multiple disabilities to be rehabilitated.

When designs serve specific populations, not only in the social sphere, but include the user from the perspective of disability, the process of creation reaches its most important contribution: this characterizes design as a tool that promotes good health.

Thus, the design process extrapolates the objectives of efficiency, effectiveness and satisfaction and achieves a new construct: functionality, considered by the World Health Organization (WHO) as a variable that is indispensible for defining the state of health itself.

In this context, virtual reality immersion, by activating the cognitive processes needed for browsing and interpreting the environment, will promote the automation of everyday activities, thus bringing about permanent changes in the subjects' mental models.

During immersion, learning overcomes the limit of the cognitive mind supported by sensory-motor corporeality, thus allowing full analysis of the subject in an environment of controlled variables.

The researcher can then define the action plans for sensory-motor action and for mental-relational action, thereby controlling a new process of self-awareness and promoting the alteration of the postural engrams within the pathology itself.

What is therefore concluded is the importance of the design process in maintaining good health and fostering functional independence, its being a decisive part of rehabilitating and reintegrating people with disabilities.

\section{References}

1. Alamri, Eid, M., Iglesias, Shirmohammadi, Saddik, A.E.: Haptic Virtual Rehabilitation Exercises for Poststroke Diagnosis. IEEE Transactions on Instrumentation and Measurement 57(9) (September 2008)

2. Alamri, Cha, El Saddik: AR-REHAB: An Augmented Reality Framework for PoststrokePatient Rehabilitation. IEEE Transactions on Instrumentation and Measurement (May 2010)

3. Antley, Slater: The Effect on Lower Spine Muscle Activation of Walking on a Narrow Beam in Virtual Reality. IEEE Transactions on Visualization and Computer Graphics 17(2) (February 2011)

4. Bohil, Alicea, Biocca: Virtual reality in neuroscience research and therapy. Nature Neuroscience 12 (December 2011)

5. Cameirão, Bermúdez, Duarte, Verschure: Virtual reality based rehabilitation speeds up functional recovery of the upper extremities after stroke: A randomized controlled pilot 
study in the acute phase of stroke using the Rehabilitation Gaming System. Restorative Neurology and Neuroscience 29, 287-298 (2011)

6. Chen, Jeng, Fung, Doong, Chuang: Psychological Benefits of Virtual Reality for Patients in Rehabilitation Therapy. Journal of Sport Rehabilitation 18, 258-268 (2009)

7. Connelly, Jia, Toro, Stoykov, Kenyon, Kamper: A Pneumatic Glove and Immersive Virtual Reality Environment for Hand Rehabilitative Training After Stroke. IEEE Transactions on Neural Systems and Rehabilitation Engineering 18(5) (October 2010)

8. Crosbie, Lennon, McGoldrick, McNeill, McDonough: Virtual reality in the rehabilitation of the arm after hemiplegic stroke: a randomized controlled pilot study. Clinical Rehabilitation 26(9), 798-806 (2012)

9. Jack, D., Boian, R., Merians, A.S., Tremaine, M., Burdea, G.C., Adamovich, S.V., Recce, M., Poizner, H.: Virtual Reality-Enhanced Stroke Rehabilitation. IEEE Transactions on Neural Systems and Rehabilitation Engineering 9(3) (September 2001)

10. Sharan, D., Ajeesh, Rameshkumar, Mathankumar, Jospin Paulina, Manjula: Virtual reality based therapy for post operative rehabilitation of children with cerebral palsy. Work 41, 3612-3615 (2012)

11. Diodato, Mraz, Baker, Graham: A Haptic Force Feedback Device for Virtual Reality-fMRI Experiments. IEEE Transactions on Neural Systems and Rehabilitation Engineering 15(4) (December 2007)

12. Galvin, Levac: Facilitating clinical decision-making about the use of virtual reality within paediatric motor rehabilitation: Describing and classifying virtual reality systems. Developmental Neurorehabilitation 14(2), 112-122 (2011)

13. Gyia, Sims, Porter, Marshall, Case: Representing older and disabled people in virtual user trials: data collection methods. Applied Ergonomics 35, 443-451 (2004)

14. Harmatz: Entering a new treatment age for mucopolysaccharidosis VI disease: a search for better markers of disease progression and response to treatment. Journal de Pediatria 84(2) (2008)

15. Kaber, Li, Clamann, Lee: Investigating Human Performance in a Virtual Reality Haptic Simulator as Influenced by Fidelity and System Latency. IEEE Transactions on Systems, Man, and Cybernetics-Part A: Systems and Humans 42(6) (November 2012)

16. Lange, Teive, Troiano, Bitencourt, Funke, Setubal, Zanis Neto, Medeiros, Werneck, Pasquini, Bonfim: Bone marrow transplantation in patients with storage diseases. Arq Neuropsiquiatr. 64(1), 1-4 (2006)

17. Ma, Hwang, Fang, Kuo, Wang, Leong, Wang: Effects of virtual reality training on functional reaching movements in people with Parkinson's disease: a randomized controlled pilot trial. Clinical Rehabilitation 25(10), 892-902 (2011)

18. Pinto, Scheartz, Puga, Vieira, Munoz, Giuliani: Prospective study of 11 Brazilian patients with mucopolysaccharidosis II. J. Pediatr. 82(4), 273-278 (2006)

19. Rebelo, Duarte, Noriega, Soares: Virtual reality in consumer product design: methods and applications. In: Karwowski, W., Soares, M.M., Stanton, N. (eds.) Human Factors and Ergonomics in Consumer Product Design, ch. 24, pp. 381-404. CRC Press, Boca Raton (2010)

20. Resnik, Etter, Klinger, Kambe: Using virtual reality environment to facilitate training with advanced upper-limb prosthesis. Journal of Rehabilitation Research \& Development 48(6) (2011)

21. Thorne, Javadpour, Hughes, Wraith, Cowie: Craniovertebral abnormalities in type VI mucopolysaccharidosis (Maroteaux-Lamy Syndrome). Neurosugery 48(4), 849-853 (2011) 
22. Ueki, Kawasaki, Ito, Nishimoto, Abe, Aoki, Ishigure, Ojika, Mouri: Development of a Hand-Assist Robot With Multi-Degrees-of-Freedom for Rehabilitation Therapy. IEEE/ASME Transactions on Mechatronics 17(1) (February 2012)

23. Viana, Lima, Cavaleiro, Alves, Souza, Feio, Leistner-Segal, Schwartz, Giugliani, Silva: Mucopolysaccharidoses in northern Brazil: Target Mutation screening and urinary glycosaminoglycan excretion in patients undergoing enzyme replacement therapy. Genetics and Molecular Biology 34(3), 410-415 (2011)

24. Vougioukas, Berlis, Kopp, Korinthenberg, Spreer, Velthoven: Neurosurgical interventions in children with Maroteaux-Lamy Syndrome. Pediatric Neurosugery 35(1) (2001)

25. Zhou, Malric, Shirmohammadi: A New Hand-Measurement Method to Simplify Calibration in CyberGlove-Based Virtual Rehabilitation. IEEE Transactions on Instrumentation and Measurement 59(10) (October 2010) 